04,05

\title{
Получение, структура, диэлектрические и магнитные свойства керамики $\mathrm{SrFe}_{2 / 3} \mathrm{~W}_{1 / 3} \mathrm{O}_{3}$
}

\author{
(C) А.В. Павленко ${ }^{1,2}$, А.В. Турик ${ }^{1}$, Л.А. Шилкина ${ }^{1}$, С.П. Кубрин ${ }^{1}$, Ю.В. Русалев ${ }^{3}$, \\ Л.А. Резниченко ${ }^{1}$, И.Н. Андрюшина ${ }^{1}$
}

${ }^{1}$ Научно-исследовательский институт фризики ЮФу, Ростов-на-Дону, Россия

${ }^{2}$ Южный научный центр РАН,

Ростов-на-Дону, Россия

${ }^{3}$ International Research Center „Smart materials“, Southern Federal University, Rostov-on-Don, Russia

E-mail: AntVPR@mail.ru

(Поступила в Редакцию 6 сентября 2017 г.)

\begin{abstract}
Методом твердофазных реакций с последующим спеканием по обычной керамической технологии получены поликристаллические образцы керамики $\mathrm{SrFe}_{2 / 3} \mathrm{~W}_{1 / 3} \mathrm{O}_{3}$ (SFWO). Рентгеноструктурный анализ показал, что при комнатной температуре керамика SFWO однофазна и обладает структурой типа перовскита с тетрагональной симметрией и параметрами: $a=3.941(9) \AA, c=3.955(6) \AA, c / a=1.0035$. При исследовании магнитных характеристик и эффекта Мессбауэра в керамике SFWO установлено, что материал является ферримагнетиком, а ионы железа находятся только в валентном состоянии $\mathrm{Fe}^{3+}$. Высказано предположение, что в SFWO в интервале температур $T=150-210^{\circ} \mathrm{C}$ при понижении температуры происходит размытый фазовый переход из кубической (параэлектрической) в тетрагональную (сегнетоэлектрическую) фазу.
\end{abstract}

Работа выполнена при финансовой поддержке МОН Минобрнауки России (проекты № 3.6371.2017/8.9, 3.6439.2017/8.9, 3.5346.2017/8.9) и гранта РФФИ № 16-32-60095 мол_а_дк.

DOI: $10.21883 /$ FTT.2018.03.45554.263

\section{1. Введение}

Мультиферроикам, в которых магнитное и электрическое упорядочения сосуществуют в широком интервале температур, уделяется все большее внимание, что обусловлено как возможностью их использования в современных устройствах [1], так и наличием в них ряда новых свойств, отсутствующих в сегнетоэлектриках и магнетиках [2]. Двойные железосодержащие перовскиты с общей химической формулой $\mathrm{AFe}_{2 / 3} \mathrm{~W}_{1 / 3} \mathrm{O}_{3}(A=\mathrm{Pb}, \mathrm{Sr}$, $\mathrm{Ba}, \mathrm{Ca})$ являются типичными представителями данного класса веществ. Впервые эти соединения были синтезированы в середине 1960-х годов [2-4], однако интенсивно начали исследоваться лишь в последние десятилетия, когда появилась необходимость создания мультиферроиков с достаточно высокими температурами магнитных и сегнетоэлектрических (СЭ) фазовых переходов (ФП) [5-8]. Как показывает анализ литературы, к настоящему времени хорошо изучены свойства лишь $\mathrm{PbFe}_{2 / 3} \mathrm{~W}_{1 / 3} \mathrm{O}_{3}$, а характеристики остальных соединений изучены фрагментарно. Более того, сведения о температурах СЭ $\left(T_{C}\right)$ и магнитного $\left(T_{M}\right)$ ФП, магнитоэлектрическом и магнитодиэлектрическом эффектах, валентностях катионов $\mathrm{Fe}$ и W зачастую неоднозначны, либо вообще отсутствуют. Так, например, согласно $[5,8] \mathrm{SrFe}_{2 / 3} \mathrm{~W}_{1 / 3} \mathrm{O}_{3}$ (SFWO) в интервале температур $-263-150^{\circ} \mathrm{C}$ обладает тетрагональной структурой с пространственной группой $I 4 / m$ и при $T<100^{\circ} \mathrm{C}$ характеризуется сосуществованием ферримагнитных и антисегнетоэлектрических свойств. Однако экспериментальное подтверждение антисегнетоэлектрического состояния в SFWO электрофизическими измерениями в настоящее времени отсутствует.

Учитывая, что SFWO является ферримагнетиком с высокими значения $T_{M}$ и $T_{C}$, благоприятствующими для использования этого соединения в качестве компонента новых мультиферроиков, представляются актуальными исследования, направленные на установление закономерностей формирования его структуры и диэлектрических характеристик в области температур $20-300^{\circ} \mathrm{C}$, чему и посвящена настоящая работа.

\section{2. Методы получения и исследования образцов}

Керамика SWFO была изготовлена методом твердофазных реакций из $\mathrm{SrCO}_{3}, \mathrm{WO}_{3}$ и $\mathrm{Fe}_{2} \mathrm{O}_{3}$ высокой степени чистоты (ч, чда) обжигом в две стадии, с промежуточным помолом, при температурах $T_{1}=1000^{\circ} \mathrm{C}$ и $T_{2}=1200^{\circ} \mathrm{C}$ и временах обжига $\tau_{1}=4 \mathrm{~h}$ и $\tau_{2}=2 \mathrm{~h}$. Режим спекания керамических заготовок SWFO составил $T_{\text {сп }}=1400^{\circ} \mathrm{C}$ и $\tau_{\text {сп }}=2.5 \mathrm{~h}$.

Рентгенофазовый (РФА) и рентгеноструктурный (РСА) анализы проводили на дифрактометре ДРОН-3 с использованием Со $K_{\alpha}$-излучения. Расчет параметров ячейки выполнен по стандартной методике [3]. Мессбауэровские спектры измерялись с помощью спектрометра 

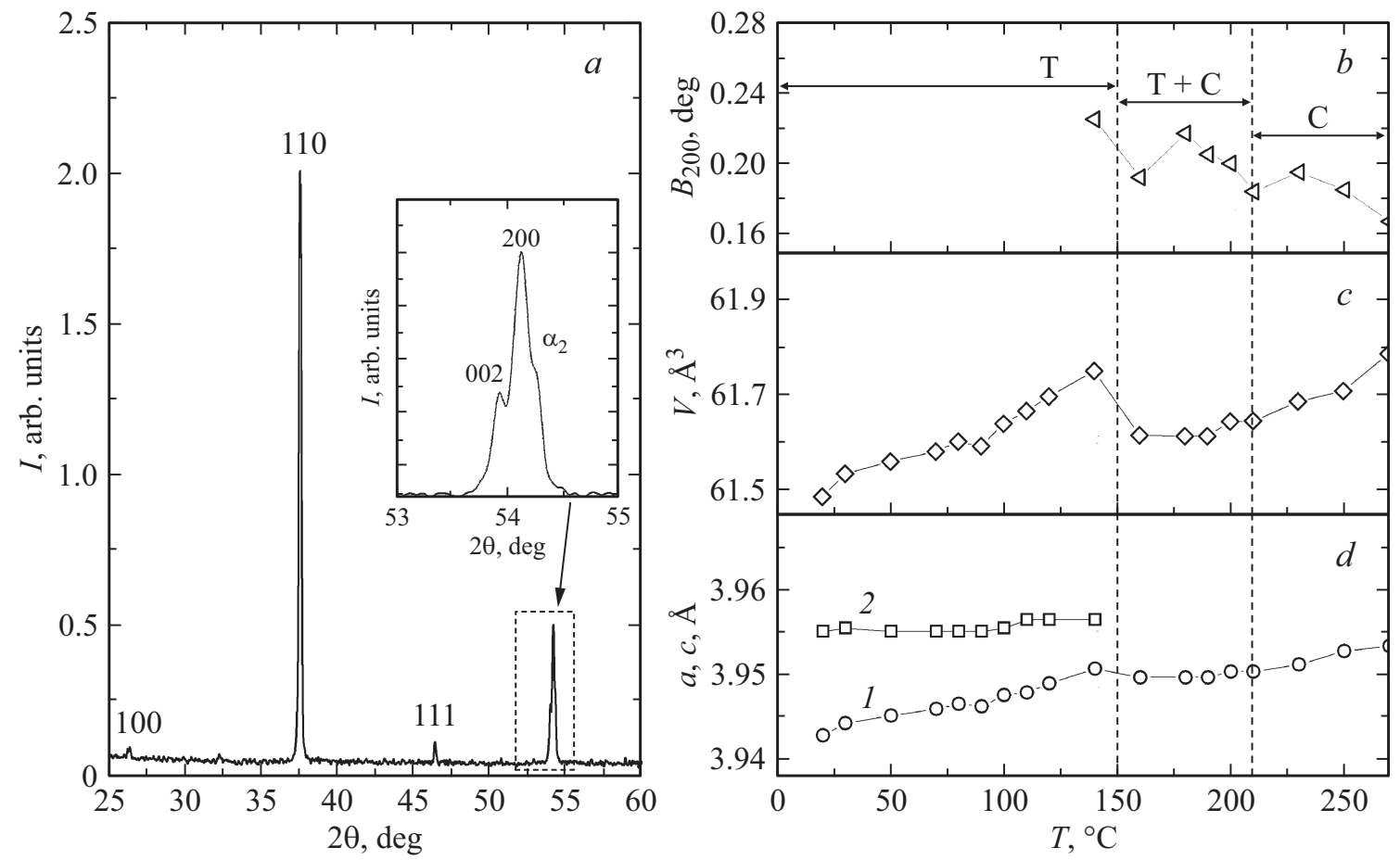

Рис. 1. $a$ - рентгенограмма керамики SWFO. $b-d-$ температурные зависимости: полуширины линии $200, B$ 200, объема, $V$, и параметров ячейки $a(1), c(2)$ соединения SWFO соответственно. Для $T=140^{\circ} \mathrm{C}$ приведена полуширина мультиплета 002,200 , аппроксимированного функцией Лоренца для одиночной линии, соответствующей неразрешенному дублету.

MS1104Еm. Источником гамма-квантов служил ${ }^{57}$ Со в матрице Rh. Модельную расшифровку спектров проводили в программе SpectrRelax. Изомерные химические сдвиги определяли относительно металлического $\alpha$-Fe.

Магнитные измерения были проведены на магнитометре Lakeshore VSM 7404. Измерения относительной диэлектрической проницаемости $\left(\varepsilon / \varepsilon_{0}\right.$, где $\left.\varepsilon_{0}=8.854 \cdot 10^{-12} \mathrm{~F} / \mathrm{m}\right)$ и тангенса угла диэлектрических потерь $(\operatorname{tg} \delta)$ в диапазоне частот $f=10^{2}-10^{5} \mathrm{~Hz}$ и удельного сопротивления $\rho$ при $T=30-300^{\circ} \mathrm{C}$ проводили с помощью LRC-метра HIOKI3522. Петли диэлектрического гистерезиса (зависимости поляризованности $P$ от электрического напряжения $U$ ) при $T \sim 30^{\circ} \mathrm{C}$ на частоте $500 \mathrm{~Hz}$ получали с помощью анализатора TFAnalizer 2000.

\section{3. Экспериментальные результаты и обсуждение}

На рис. 1, $a$ приведена рентгенограмма керамики SFWO, снятая при комнатной температуре. Установлено, что объекту свойственна структура типа перовскита с тетрагональной (Т) симметрией и параметрами: $a=3.941(9) \AA, c=3.955(6) \AA, c / a=1.0035$. При угле $2 \theta=32.3^{\circ}$ идентифицирована слабая сверхструктурная линия, указывающая на удвоение параметров перовскитной ячейки, что свидетельствует о частичной локальной упорядоченности в расположении катионов $\mathrm{Fe}$ и W.
Рентгенографическое исследование в интервале температур $20 \leq T \leq 300^{\circ} \mathrm{C}$ показало, что кристаллическая решетка SFWO сохраняет T симметрию в интервале $20 \leq T<150^{\circ} \mathrm{C}$, имеет кубическую симметрию (С) при $T>225^{\circ} \mathrm{C}$, а в области температур $150<T \leq 225^{\circ} \mathrm{C}$ фазы Т и $\mathrm{C}$ сосуществуют.

На рис. $1, b-c$ представлены зависимости от температуры параметров $a, c$, объема $(V)$ и полуширины рентгеновской линии $(200)_{\kappa}, B_{200}$. Видно, что эти зависимости носят немонотонный характер. При $T=90-100^{\circ} \mathrm{C}$ изменяются наклоны $a(T)$ и $V(T)$, связанные по-видимому, с магнитным фазовым переходом [5]. При $T=140-150^{\circ} \mathrm{C}$ обнаруживаются скачки объема $\Delta V=-0.14 \AA^{3}$ и $B_{200}$, а в интервале $T=160-200^{\circ} \mathrm{C}$ имеет место постоянство параметра и объема ячейки (инварный эффект), обусловленное размытым ФП из Т в С фазу (т. е. в этой области Т в С фазы сосуществуют, а увеличение температуры приводит к изменению соотношения фаз), аналогично тому, как это имело место в керамике $\mathrm{PbFe}_{1 / 2} \mathrm{Nb}_{1 / 2} \mathrm{O}_{3}$ [9]. В С-фазе $B_{200}$ продолжает медленно убывать, а $a$ и $V$, вследствие теплового расширения, увеличиваться.

Результаты исследования эффекта Мессбауэра в керамике SWFO приведены на рис. 2 и в таблице. Мессбауэровский спектр представляют собой суперпозицию трех Зеемановских секстетов (рис. 2), соответствующих трем неэквивалентным магнитным состояниям ионов $\mathrm{Fe}^{3+}$. Параметры секстетов приведены в таблице. Величины изомерных сдвигов секстетов спектра образца SFWO 
Параметры мессбауэровского спектра комнатной температуры образца $\mathrm{SrFe}_{2 / 3} \mathrm{~W}_{1 / 3} \mathrm{O}_{3}$

\begin{tabular}{l|c|c|c|c|c}
\hline Компонента & $\delta \pm 0.02, \mathrm{~mm} / \mathrm{s}$ & $\varepsilon \pm 0.02, \mathrm{~mm} / \mathrm{s}$ & $H \pm 1, \mathrm{kOe}$ & $A \pm 1, \%$ & $G \pm 0.02, \mathrm{~mm} / \mathrm{s}$ \\
\hline Секстет 1 & 0.38 & -0.02 & 380 & 25 & 0.79 \\
Секстет 2 & 0.39 & -0.01 & 348 & 58 & 1.24 \\
Секстет 3 & 0.39 & -0.01 & 300 & 17 & 0.83
\end{tabular}

Примечание $. \delta-$ изомерный сдвиг, $\varepsilon-$ квадрупольное смещение, $H-$ сверхтонкое магнитное поле на ядрах ${ }^{57}$ Fе, $A-$ площадь компонент спектра, $G$ - ширина линий спектра.

примерно равны друг другу и соответствуют ионам $\mathrm{Fe}^{3+}$ в октаэдрическом кислородном окружении [10].

На рис. 3 приведены петли магнитного гистерезиca $M(H)$ керамик SWFO, измеренные при $T=30^{\circ} \mathrm{C}$. Зависимость $M(H)$ образца SWFO имеет свойственную ферримагнетикам вытянутую форму, а величины остаточной намагниченности и коэрцитивного поля составили $0.052 \mathrm{emu} / \mathrm{g}$ и 598 Ое соответственно. Появление ферримагнитных свойств в SWFO, согласно [5,7], может быть обусловлено двумя причинами: во-первых, существованием в его структуре двух или более типов позиций для атомов железа и частичным упорядочением катионов $\mathrm{Fe}^{3+}$ и $\mathrm{W}^{6+}$, во-вторых, появлением катионов $\mathrm{Fe}^{2+}$. Результаты РСА и эффекта МБ свидетель-

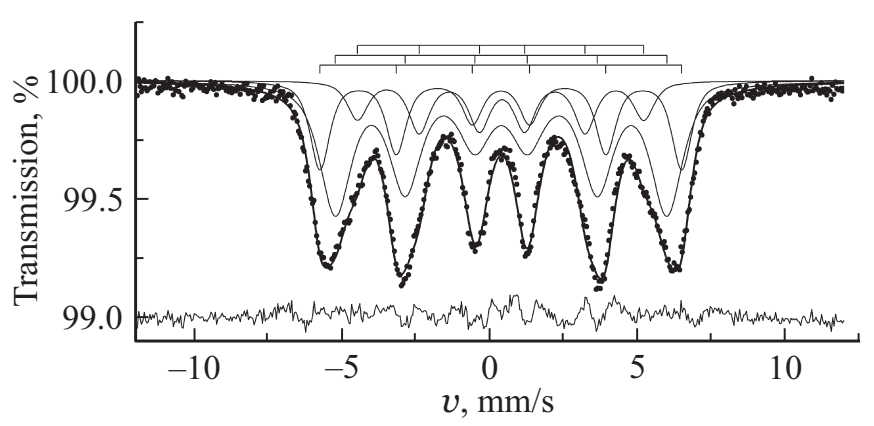

Рис. 2. Мессбауэровский спектр образца SWFO, измеренный при комнатной температуре.

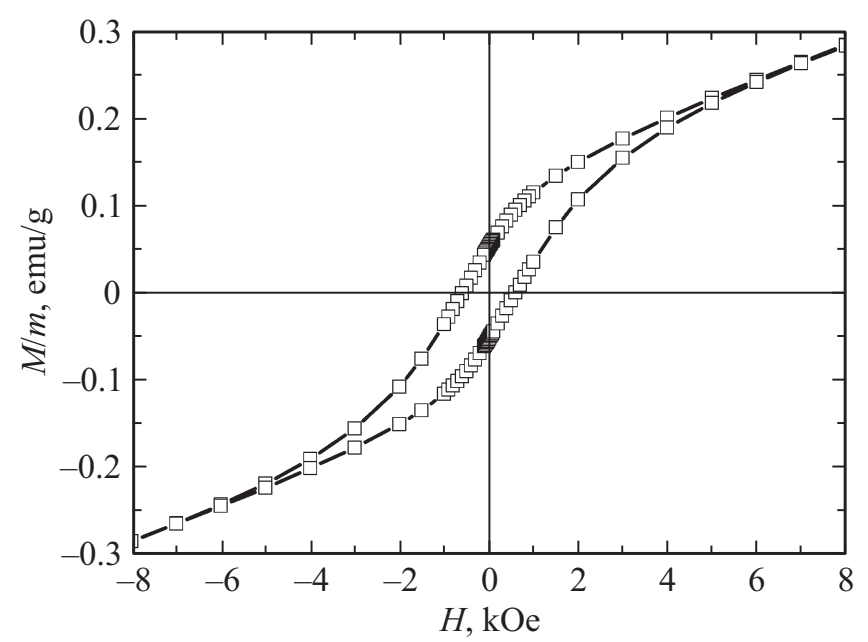

Рис. 3. Зависимости $M(H)$ керамики SWFO при $T=30^{\circ} \mathrm{C}$.

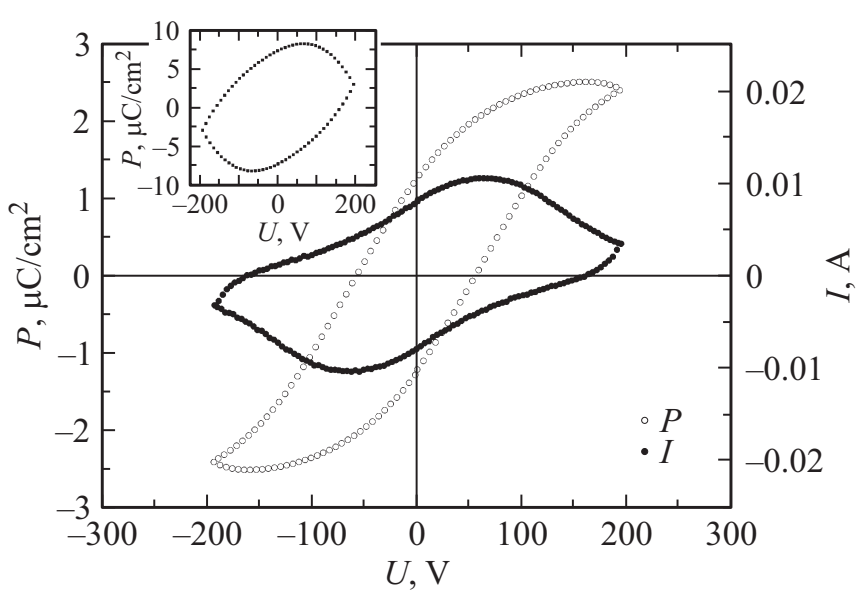

Рис. 4. Зависимости $P(U)$ и $I(U)$ керамики SWFO при $T=30^{\circ} \mathrm{C}$, измеренные в режиме учета компенсации токов утечки. На вставке - зависимость $P(U)$ без учета компенсации токов утечки.

ствуют в пользу того, что в нашем случае реализуется первая ситуация.

B SWFO зависимость $P(U)$ (рис. 4, вставка), имела эллипсоидальную форму, что обусловлено низким сопротивлением керамики $\left(\rho=5 \cdot 10^{5} \Omega \cdot \mathrm{m}\right)$. С целью исключения при измерении петель диэлектрического гистерезиса вкладов токов утечки был использован режим измерения анализатора TFAnalizer 2000. Это позволило фиксировать характерные для СЭ кривые $P(U)$ и $I(U)$.

На рис. 5 приведены зависимости $\varepsilon / \varepsilon_{0}(T)$ и $\operatorname{tg} \delta(T)$ $\left(\varepsilon_{0}\right.$ - электрическая постоянная) керамики SWFO. При $T=30^{\circ} \mathrm{C}$ в SWFO имеет место максимальная для исследованного диапазона температур дисперсия диэлектрической проницаемости $\Delta \varepsilon=\left(\varepsilon\left(f=10^{2} \mathrm{~Hz}\right)\right.$ $\left.-\varepsilon\left(f=10^{5} \mathrm{~Hz}\right)\right) / \varepsilon\left(f=10^{2} \mathrm{~Hz}\right)$ (рис. 6). С повышением температуры наблюдается уменьшение $\Delta \varepsilon$ и формирование в области магнитного ФП, согласно [5], слабовыраженных максимумов $\varepsilon / \varepsilon_{0}(T)$ и $\operatorname{tg} \delta(T)$, связанных с проявлением магнитоэлектрического взаимодействия. Дальнейший рост температуры сопровождается увеличением $\varepsilon / \varepsilon_{0}$ и $\operatorname{tg} \delta$ и формированием при $T=150-220^{\circ} \mathrm{C}$ частотно-зависимых размытых максимумов $\varepsilon / \varepsilon_{0}\left(T=T_{m}\right)$, связанных, согласно данным РСА, с размытым ФП из тетрагональной в кубическую фазу. Зависимость $T_{m}(f)$ аппроксимирована соотношением 


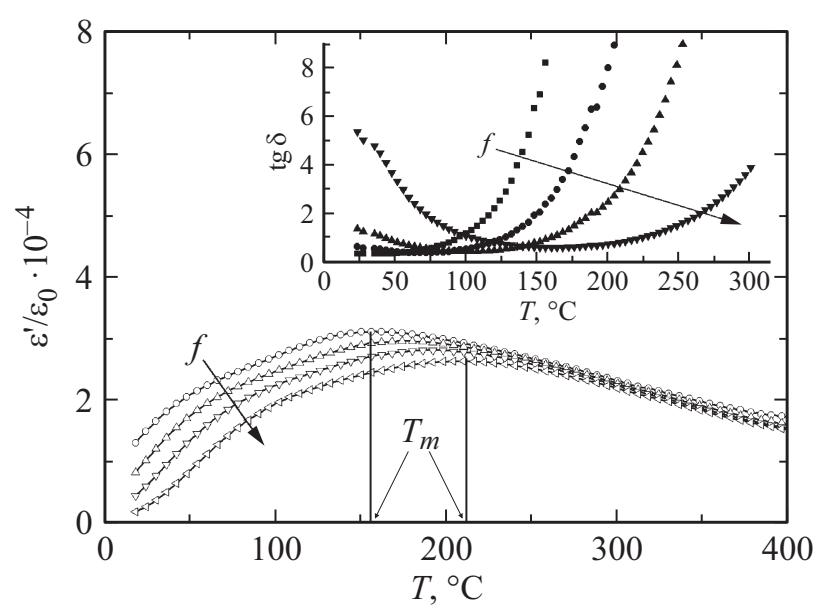

Рис. 5. Зависимости $\varepsilon / \varepsilon_{0}(T)$ и $\operatorname{tg} \delta(T)$ керамики и SWFO при $T=30-300^{\circ} \mathrm{C}$ и $f=10^{3}-10^{5} \mathrm{~Hz}$, снятые в режиме охлаждения.

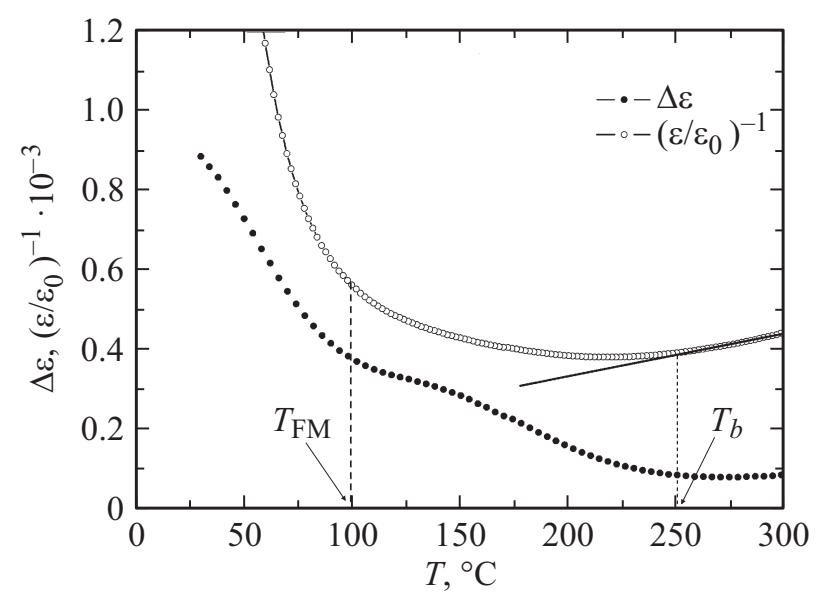

Рис. 6. Зависимости $\left(\varepsilon / \varepsilon_{0}\right)^{-1}(T)$ и $\Delta \varepsilon(T)$ керамики SWFO. $T_{\mathrm{FM}}$ - температура магнитного фазового перехода согласно [5].

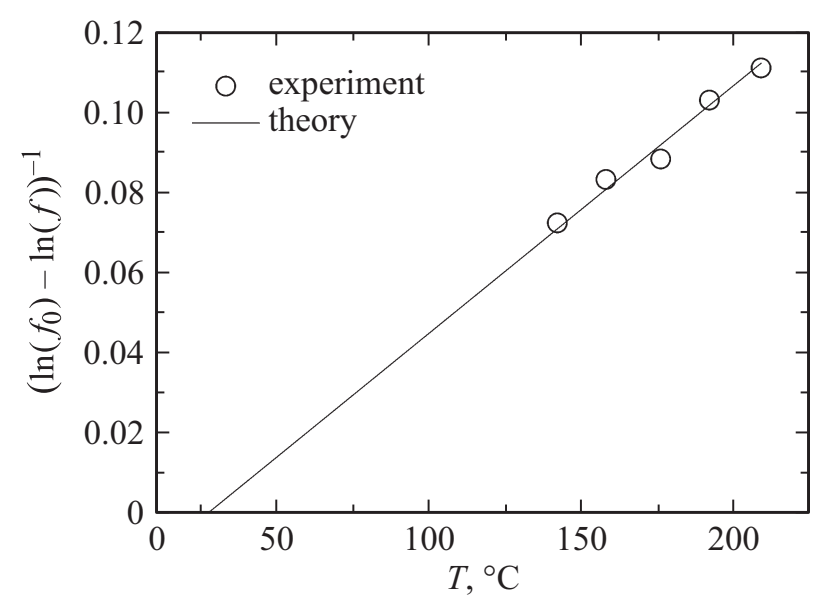

Рис. 7. Зависимость $\left(\ln \left(f_{0}\right)-\ln (f)\right)^{-1}$ от $T_{m}$ в керамике SWFO, иллюстрирующая выполнение законы Фогеля-Фулчера. Светлые маркеры - эксперимент, сплошная линия — расчет.
Фогеля-Фулчера (рис. 7)

$$
f=f_{0} \exp \left(E_{\text {act }} /\left(k\left(T_{m}-T_{f}\right)\right)\right),
$$

где $f_{0}$ - частота попыток преодоления потенциального барьера $E_{\text {act }}, k$ - постоянная Больцмана, $T_{f}$ - температура Фогеля-Фулчера, интерпретируемая как температура „статического замораживания“ электрических диполей. Рассчитанное значение $E_{\text {act }} \approx 0.16 \mathrm{eV}$ характерно для сегнетоэлектриков-релаксоров, значение $T_{f} \approx 10^{\circ} \mathrm{C}$.

Рассчитанное значение температуры Бернса $\left(T_{b}\right)$ в керамике SWFO (рис. 6) составило $\sim 250^{\circ} \mathrm{C}$ и более чем на $70^{\circ} \mathrm{C}$ превышало $T_{m}$. Последнее говорит о том, что кластеры тетрагональный фазы исчезают лишь при $T \sim 250^{\circ} \mathrm{C}$, что и обусловливает фиксируемое именно в этой области температур уменьшение $B_{200}$ (рис. 2) и минимальные значения $\Delta \varepsilon$ (рис. 6). В интервале $250-300^{\circ} \mathrm{C}$ формируется практически бездисперсный участок $\varepsilon / \varepsilon_{0}(T)$.

Таким образом, результаты исследований зависимостей $P(U), \varepsilon / \varepsilon_{0}(T, f)$ и $\operatorname{tg} \delta(T, f)$ в керамике SWFO, так же как и в $\mathrm{PbF}_{2 / 3} \mathrm{~W}_{1 / 3} \mathrm{O}_{3}$ [11], свидетельствуют о том, что при понижении температуры происходит размытый ФП из параэлектрической (С) фазы в сегнетоэлектрическую (Т), что противоречит результатам работы [5], в которой предположено, что SFWO в тетрагональной фазе является антисегнетоэлектриком. Так как диэлектрических измерений в [5] не проводилось, достаточно сложно провести сопоставление результатов диэлектрических измерений. Скорее всего, наблюдаемые расхождения связаны с различными технологиями получения объектов, свойства которых, как это показано на примере керамики $\mathrm{BaF}_{2 / 3} \mathrm{~W}_{1 / 3} \mathrm{O}_{3}$ [7], могут сильно зависеть от термодинамической предыстории (условий приготовления) образцов.

\section{4. Выводы}

Установлено, что керамика SFWO при комнатной температуре является однофазной (тетрагональная симметрия), с признаками локальной упорядоченности в расположении катионов $\mathrm{Fe}$ и $\mathrm{W}$, и обладает сегнетоэлектрическими и ферримагнитными свойства. Ионы железа в объекте, согласно данным мессбауэровской спектроскопии, находятся только в валентном состоянии $\mathrm{Fe}^{3+}$ в трех неэквивалентных магнитных состояниях.

Рентгенографическое исследование керамики SWFO показало, что кристаллическая решетка SFWO в интервале температур $20 \leq T<150^{\circ} \mathrm{C}$ имеет тетрагональную симметрию, при $T>225^{\circ} \mathrm{C}-$ кубическую, а в области температур $150<T \leq 225^{\circ} \mathrm{C}$ эти фазы сосуществуют.

При исследовании диэлектрических характеристик предположено, что SFWO можно отнести к сегнетоэлектрикам-релаксорам, в которых при $150-220^{\circ} \mathrm{C}$ протекает размытый фазовый переход из сегнетоэлектрического (Т-фаза) в параэлектрическое (С-фаза) состояние. 
Полученные в работе результаты могут быть использованы при разработке мультиферроидных структур на базе двойных железо-содержащих перовскитов.

\section{Список литературы}

[1] T. Kimura, T. Goto, H. Shintani, K. Ishizaka, T. Arima, Y. Tokura. Nature 426, 55 (2003).

[2] А.П. Пятаков, А.К. Звездин. УФН 182, 6, 593 (2012).

[3] Е.Г. Фесенко. Семейство перовскита и сегнетоэлектричество. Атомиздат, М. (1972). 248 с

[4] G. Blasse. J. Inorg. Nucl. Chem. 27, 993 (1965).

[5] S.A. Ivanov, S.G. Eriksson, R. Tellgren, H. Rundlof. Mater. Res. Bull. 36, 2585 (2001).

[6] S.A. Ivanov, S.G. Eriksson, R. Tellgren, H. Rundlof. Mater. Res. Bul. 39, 615 (2004).

[7] S.A. Ivanov, S.-G. Eriksson, R. Tellgren, H. Rundlof, P. Nordblad, J. Eriksen. J. Solid State Chem. 179, 2645 (2006).

[8] M. del C. Viola, M.S. Augsburger, R.M. Pinacca, J.C. Pedregosa, R.E. Carbonio, R.C. Mercaderc. J. Solid State Chem. 175, $252(2003)$.

[9] А.В. Павленко, Л.А. Шилкина, Л.А. Резниченко. Кристаллография 56, 4, 729 (2011).

[10] F. Menil. J. Phys. Chem. Solids 46, 7, 763 (1985).

[11] Z.G. Ye, H. Schmid. Ferroelectrics 162, 119 (1994). 